\title{
Management of persistent dry cough
}

\author{
Finbarr O’Connell
}

Cough is probably the commonest symptom affecting humans, with prevalence rates variously reported within the range 5-40\%. ${ }^{1}$ Acute or short lived cough, which most commonly occurs in association with viral upper respiratory infection, is of little consequence, usually resolving in a matter of days. Persistent cough, however, may be associated with considerable morbidity including sleep loss, exhaustion, irritability, urinary stress incontinence, cough syncope, impaired performance in daily activities, and considerable social disability. Rib fractures, pneumothorax, pneumomediastinum, and subcutaneous emphysema have been reported in severe cases. ${ }^{2}$ Persistent productive cough is usually due to cigarette smoking, chronic bronchitis or bronchiectasis, and diagnosis is usually straightforward. Persistent dry cough poses a greater diagnostic and therapeutic challenge.

When clinical evaluation and basic investigations such as spirometry and chest radiography are normal, the cause of persistent dry cough may be elusive and such patients pose considerable difficulty in the clinic. Thorough systematic investigation is both necessary and worthwhile, necessary because the patient may present with cough alone without any typical features of the underlying cause, and worthwhile because treatment of the cause usually leads to resolution of the cough. ${ }^{3}$ Several studies have shown that the only underlying diagnoses commonly found on systematic investigation are asthma, gastro-oesophageal reflux (GOR), and post-nasal drip (PND). ${ }^{3-9}$ This has been confirmed again by McGarvey et al in this issue of Thorax..$^{10}$ Using a rigorously applied diagnostic protocol, they identified one or more of these three conditions as the cause of cough in $35(82 \%)$ of 43 patients presenting with persistent cough as their sole respiratory symptom.

Although carried out in a relatively small number of patients, their paper serves to highlight several important points in the management of persistent dry cough. Firstly, the history alone is entirely unreliable for diagnosis as many patients have a clinically "silent" potential underlying cause of cough. Also, patients may have features in the history suggestive of a specific diagnosis but fail to respond to specific treatment for that condition. Investigation may suggest an alternative diagnosis and the positive predictive value of the history alone for a specific underlying condition was only $40-56 \%$.

Secondly, the most valuable investigations for asthma, GOR and PND, respectively, are bronchial provocation (with histamine or methacholine), 24 hour oesophageal $\mathrm{pH}$ monitoring, and combined formal ENT assessment/CT sinuses. Some or all of these investigations may not be readily available to many clinicians, who may thus have to choose between referral to specialist centres with the necessary diagnostic facilities and empirical trials of treatment for potential underlying conditions. While the former approach is preferable, the latter may be chosen for various reasons and, if so, empirical treatment should be comprehensive and continued for at least two months. Their study is unique in having applied these investigations in all patients, allowing accurate determination of positive predictive values.

Thirdly, where investigation suggests a specific diagnosis, the cough may fail to respond to intensive and prolonged treatment for that condition. Using successful response to specific treatment as the final criterion for diagnosis, positive predictive values for the above investigations were $63-88 \%$. As all patients did not receive empirical treatment for each possible underlying condition, accurate negative predictive values could not be determined and are not reported. However, it is generally accepted-as the authors suggest and as Irwin has previously reported ${ }^{4}$ - that bronchial provocation and 24 hour oesophageal $\mathrm{pH}$ monitoring, when negative, effectively rule out asthma and GOR, respectively, as the cause of cough. Empirical treatment for asthma and GOR is therefore unnecessary where these investigations are available and prove negative.

Fourthly, in eight (19\%) of 43 patients two underlying conditions were found, similar to the $26 \%$ with multiple aetiologies previously reported. ${ }^{4}$ This emphasises the importance of investigation for further underlying conditions when the cough responds partially or fails to respond to a specific treatment.

Fifthly, while the capsaicin cough challenge data are not essential to the central clinical message of the study, they confirm the previous observation that patients with dry cough show enhanced capsaicin sensitivity which normalises with successful treatment of the cough. ${ }^{9}$ This is true for all treatment groups and confirms that enhanced sensitivity of afferent cough nerves is an important pathogenetic mechanism in patients with persistent dry cough, regardless of the specific aetiology. The authors correctly comment that capsaicin challenge provides a useful research tool for measurement of change, but has little value in routine clinical practice because of non-specificity with regard to aetiology.

Finally, the study by McGarvey et al confirms that, in a significant proportion (18\%) of patients presenting with persistent dry cough, no underlying cause of the cough may be found despite complete investigation and trials of intensive empirical treatment for GOR and PND. This is in contrast with the studies reported by Irwin et $a l^{3}{ }^{4}$ but entirely in keeping with other reports, ${ }^{7}$ and in one recent prospective study in 87 patients no underlying cause was found in $31 \% .{ }^{9}$ For these patients with idiopathic persistent dry cough, specific treatment of the underlying cause is, by definition, impossible and treatment must rely on antitussive therapy. Cough sensitivity to inhaled tussigens such as capsaicin is also enhanced in these patients, and it is tempting to suggest that an ideal antitussive drug would reduce this altered sensitivity to normal, without significant adverse effects. Despite significant advances in our understanding of the pathogenesis of cough, such a drug is not yet available. Recent evidence suggests that calcitonin gene-related peptide and substance $\mathrm{P}$ may play a role in the pathogenesis of idiopathic persistent dry cough, and antagonists of these compounds may prove useful as potential antitussive treatment in future studies. ${ }^{11}$

Meanwhile, patients presenting with persistent dry cough are best served by thorough investigation, particularly for asthma, GOR, and PND using protocols such as those initially described by Irwin, ${ }^{3}{ }^{4}$ subsequently by others, ${ }^{9}$ and in this issue of Thorax by McGarvey and co-authors. ${ }^{10}$ 
1 Fuller RW, Jackson DM. Physiology and treatment of cough. Thorax 1990;45:425-30.

2 Leith DE, Butler JP, Sneddon SL, et al. Cough. In: Handbook of physiology. The respiratory system. Control of breathing. Section 1, Volume II, part 1. Bethesda: American Physiological Society, 1986: 315-36.

3 Irwin RS, Corrao WM, Pratter MR. Chronic persistent cough in the adult: the spectrum and frequency of causes and successful outcome of specific therapy. Am Rev Respir Dis 1981;123:413-7.

4 Irwin RS, Curley FJ, French CL. Chronic cough. The spectrum and frequency of causes, key components of the diagnostic evaluation, and outcome of specific therapy. Am Rev Respir Dis 1990;141:640-7.

5 Choudry NB, Fuller RW. Sensitivity of the cough reflex in patients with chronic cough. Eur Respir f 1992;5:296-300.

6 Hollinger LD. Chronic cough in infants and children. Laryngoscope 1986;96:316-22.
7 Poe RH, Harder RV, Israel RH, et al. Chronic persistent cough. Experience in diagnosis and outcome using an anatomic diagnostic protocol. Chest in diagnosis and

8 Stone RA, Barnes PJ. Patients with cough have increased sensitivity to low chloride and capsaicin solutions compared to normal and asthmatic subjects (abstract). Respir Med 1992;86:78.

9 O'Connell F, Thomas VE, Pride NB, et al. Capsaicin cough sensitivity decreases with successful treatment of chronic cough. Am $\mathcal{F}$ Respir Crit Care Med 1994;150:374-80.

10 McGarvey LPA, Heaney LG, Lawson JT, et al. Evaluation and outcome of patients with chronic non-productive cough using a comprehensive diagnostic protocol. Thorax 1998;53:738-43.

11 O'Connell F, Springall DR, Moradoghli-Haftvani A, et al. Abnormal intraepithelial airway nerves in persistent unexplained cough. Am f Respir Crit Care Med 1995;152:2068-75. 